\title{
The local well-posedness and stability to a nonlinear generalized Degasperis-Procesi equation
}

Jing Chen ${ }^{*}$ and Rui Li

\section{"Correspondence:}

mychenjing2007@sina.com

'School of Science, Southwest

University of Science and

Technology, Mianyang, 621000,

China

Full list of author information is

available at the end of the article

\begin{abstract}
A nonlinear generalized Degasperis-Procesi equation is investigated. The local well-posedness of a strong solution for the equation in the Sobolev space $H^{5}(R)$ with $s>\frac{3}{2}$ is established. The $L^{1}(R)$ stability is obtained under certain assumptions on the initial data.
\end{abstract}

MSC: $35 \mathrm{G} 25 ; 35 \mathrm{~L} 05$

Keywords: nonlinear equation; Sobolev space $H^{5}(R) ; L^{1}(R)$ stability

\section{Introduction}

Degasperis and Procesi [1] investigated a family of third order dispersive nonlinear equations

$$
u_{t}+\alpha_{0} u_{x}+\gamma u_{x x x}-\beta^{2} u_{t x x}=\left(\alpha_{1} u^{2}+\alpha_{2} u_{x}^{2}+\alpha_{3} u u_{x x}\right)_{x}
$$

where constants $\alpha_{i}(i=0,1,2,3), \beta$ and $\gamma \in R$.

Applying the method of asymptotic integrability to Eq. (1), it is found in [1] that only three equations satisfy asymptotic integrability conditions: the KdV equation, the Camassa-Holm $(\mathrm{CH})$ equation and one new equation of the form

$$
u_{t}+u_{x}+6 u u_{x}+u_{x x x}-\beta^{2}\left(u_{t x x}+\frac{9}{2} u_{x} u_{x x}+\frac{3}{2} u u_{x x x}\right)=0
$$

which can be transformed to the dispersionless form

$$
u_{t}-u_{t x x}+4 u u_{x}=3 u_{x} u_{x x}+u u_{x x x}, \quad t>0, x \in R
$$

Equation (3) is called Degasperis-Procesi (DP) equation which represents a model for shallow water dynamics, and its asymptotic accuracy is similar to the $\mathrm{CH}$ equation.

Here, we set the coefficients of Eq. (1) $\gamma=-\alpha_{0} \beta^{2}, \alpha_{2}=\alpha_{3}$ and $\beta>0$. The objective of this work is to study the following nonlinear equation:

$$
u_{t}+\alpha_{0} u_{x}-\beta^{2}\left(\alpha_{0} u_{x x x}+u_{t x x}\right)=2 \alpha_{1} u u_{x}+3 \alpha_{2} u_{x} u_{x x}+\alpha_{2} u u_{x x x} .
$$

Obviously, DP equation (3) is the special case of Eq. (4).

(c) 2015 Chen and Li. This article is distributed under the terms of the Creative Commons Attribution 4.0 International License (http://creativecommons.org/licenses/by/4.0/), which permits unrestricted use, distribution, and reproduction in any medium, provided you give appropriate credit to the original author(s) and the source, provide a link to the Creative Commons license, and indicate if changes were made. 
Degasperis et al. [2] proved the formal integrability of Eq. (3), derived the infinite sequence of conserved quantities with a bi-Hamiltonian structure and obtained the existence of the non-smooth solutions by constructing a Lax pair. Lenells [3] classified all weak traveling wave solutions of Eq. (3), while Coclite and Karlsen [4] established the well-posedness of periodic weak solutions for Eq. (3) and studied their long-time asymptotic behavior. It was shown in [5] that several numerical schemes were constructed and proved that they converged to weak solutions of Eq. (3). Escher et al. [6] discussed several qualitative properties of the DP equation. The existence and uniqueness of global weak solutions for Eq. (3) have been established provided that the initial data satisfy appropriate conditions in [6].

In fact, the KdV equation modeling weakly nonlinear unidirectional long waves and the $\mathrm{CH}$ equation modeling the unidirectional shallow water waves have been extensively investigated (see [7-11]). For other approaches to study related partial differential equations, the reader is referred to [12-20] and the references therein.

In this paper, assuming that the initial value $u(0, x)$ of Eq. (4) belongs to $H^{s}(R)\left(s>\frac{3}{2}\right)$, we will prove the existence and uniqueness of the local solution for Eq. (4) in the space $C\left([0, \infty) ; H^{s}(R)\right) \cap C^{1}\left([0, \infty) ; H^{s-1}(R)\right)$ by using the Kato theorem (see [20]) and use the approaches presented in Kruzkov [19] to establish the $L^{1}(R)$ local stability of the solution for this nonlinear equation (4). From our knowledge, Eq. (4) has not been discussed in the literature.

In the following, we will give the conservation law in some case for Eq. (4) in Section 2. The proof of existence for the local strong solution and its stability to Eq. (4) are given in Section 3 and Section 4, respectively. For simplicity, we let $c$ denote any positive constants.

\section{Preliminaries}

Applying the operator $\Lambda^{-2}=\left(1-\beta^{2} \partial_{x}^{2}\right)^{-1}$ to Eq. (4), we have

$$
u_{t}+\left(\alpha_{0}+b_{1} u\right) u_{x}+b_{2} \Lambda^{-2} \partial_{x} u^{2}=0
$$

where $b_{1}=\frac{\alpha_{2}}{\beta^{2}}, b_{2}=-\left(\alpha_{1}+\frac{\alpha_{2}}{2 \beta^{2}}\right)$.

Here we consider the Cauchy problem of Eq. (4)

$$
\left\{\begin{array}{l}
u_{t}+\alpha_{0} u_{x}-\beta^{2}\left(\alpha_{0} u_{x x x}+u_{t x x}\right)=2 \alpha_{1} u u_{x}+3 \alpha_{2} u_{x} u_{x x}+\alpha_{2} u u_{x x x} \\
u(0, x)=u_{0}(x)
\end{array}\right.
$$

which is equivalent to the problem

$$
\left\{\begin{array}{l}
u_{t}+\left(\alpha_{0}+b_{1} u\right) u_{x}+b_{2} \Lambda^{-2} \partial_{x} u^{2}=0 \\
u(0, x)=u_{0}(x)
\end{array}\right.
$$

Lemma 2.1 Let $y_{1}=u-\beta^{2} \partial_{x}^{2} u$ and $\bar{y}=\left(1-\beta^{2} \partial_{x}^{2}\right)^{-1} u$. The solutions of Eq. (4) in case $\alpha_{1}=$ $-\frac{\alpha_{2}}{2 \beta^{2}}$ conform with the following conservation law:

$$
\int_{R} y_{1} \bar{y} d x=\int_{R}|\hat{u}(t, \xi)|^{2} d \xi=\int_{R}\left|\hat{u}_{0}(\xi)\right|^{2} d \xi
$$

where $u_{0}(x)=u(0, x), \hat{u}(t, \xi)$ is the Fourier transform of $u(t, x)$ with respect to $x$. 
Proof For $y_{1}=u-\beta^{2} \partial_{x}^{2} u, \bar{y}=\left(1-\beta^{2} \partial_{x}^{2}\right)^{-1} u$, we have $\frac{\partial y_{1}}{\partial t}=u_{t}-\beta^{2} \partial_{x}^{2} u_{t x x}$ and $u=\bar{y}-\beta^{2} \bar{y}_{x x}$. If $\alpha_{1}=-\frac{\alpha_{2}}{2 \beta^{2}}$, it has

$$
\begin{aligned}
\frac{d}{d t} \int_{R} y_{1} \bar{y} d x & =2 \int_{R} \frac{\partial y_{1}}{\partial t} \bar{y} d x \\
& =2 \int_{R}\left(-\alpha_{0} u_{t}+\alpha_{0} \beta^{2} u_{x x x}+2 \alpha_{1} u u_{x}+3 \alpha_{2} u_{x} u_{x x}+\alpha_{2} u u_{x x x}\right) \bar{y} d x \\
& =2 \int_{R} \alpha_{0}\left(u \bar{y}_{x}-\beta^{2} u \bar{y}_{x x x}\right)-\left(\alpha_{1} u^{2} \bar{y}_{x}+\frac{\alpha_{2}}{2} u^{2} \bar{y}_{x x x}\right) d x \\
& =2 \int_{R}\left(\alpha_{0} u-\alpha_{1} u^{2}\right) u_{x} d x \\
& =0 .
\end{aligned}
$$

This completes the proof of Lemma 2.1.

\section{Existence of the local solution for problem (6)}

Firstly, we need to state some notions. Let $H^{s}(R)$ (where $s$ is a real number) denote the Sobolev space with the norm defined by

$$
\|h\|_{H^{s}}=\left(\int_{R}\left(1+|\xi|^{2}\right)^{s}|\hat{h}(t, \xi)|^{2}\right)^{\frac{1}{2}}<\infty
$$

where $\hat{h}(t, \xi)=\int_{R} e^{-i x \xi} h(t, x) d x$. For $T>0$ and $s \geq 0$, we let $C\left([0, \infty) ; H^{s}(R)\right)$ denote the Fréchet space of all continuous $H^{s}$-valued functions on $[0, T)$.

We introduce the abstract quasi-linear evolution equation

$$
\left\{\begin{array}{l}
\frac{d \phi}{d t}+Q(\phi) \phi=g(\phi), \quad t \geq 0 \\
\phi(0)=\phi_{0}
\end{array}\right.
$$

Let $X$ and $Y$ be Hilbert spaces, where $Y$ is continuously and densely embedded in $X$, and $W: Y \rightarrow X$ be a topological isomorphism. We define $L(Y, X)$ to be the space of all bounded linear operators from $Y$ to $X$. We denote $L(X, X)$ by $L(X)$. Note that $\rho_{1}, \rho_{2}, \rho_{3}$ and $\rho_{4}$ in the following depend on $\max \left\{\|y\|_{Y},\|z\|_{Y}\right\}$.

(I) $Q(y) \in L(Y, X)$ for $y \in X$ with

$$
\|(Q(y)-Q(z)) w\|_{X} \leq \rho_{1}\|y-z\|_{X}\|w\|_{Y}, \quad y, z, w \in Y,
$$

$Q(y) \in G(X, 1, \alpha)(\alpha>0)$ and uniformly on bounded sets in $Y$.

(II) $W Q(y) W^{-1}=Q(y)+\bar{Q}(y)$, in which $\bar{Q} \in L(X)$ is bounded and uniformly on bounded sets in $Y$ and

$$
\|(\bar{Q}(y)-\bar{Q}(z)) w\|_{X} \leq \rho_{2}\|y-z\|_{X}\|w\|_{Y}, \quad y, z \in Y, w \in X .
$$

(III) $g: Y \rightarrow Y$ extends to a map from $X$ into $X$, is bounded on bounded sets in $Y$ and satisfies

$$
\begin{aligned}
& \|g(y)-g(z)\|_{Y} \leq \rho_{3}\|y-z\|_{Y}, \quad y, z \in Y, \\
& \|g(y)-g(z)\|_{X} \leq \rho_{4}\|y-z\|_{X}, \quad y, z \in Y .
\end{aligned}
$$


Kato theorem ([20]) Assume that (I), (II) and (III) hold. If $\phi_{0} \in Y$, there is a maximal $T>0$ depending only on $\left\|\phi_{0}\right\|_{Y}$ and a unique solution u to problem (9) such that

$$
\phi=\phi\left(\cdot, \phi_{0}\right) \in C([0, T) ; Y) \cap C^{1}([0, T) ; X) .
$$

Moreover, the map $\phi_{0} \rightarrow \phi\left(\cdot, \phi_{0}\right)$ is a continuous map from $Y$ to the space $C([0, T) ; Y) \cap$ $C^{1}([0, T) ; X)$.

For problem (7), we set $Q(u)=\left(\alpha_{0}+b_{1} u\right) \partial_{x}, Y=H^{s}(R), X=H^{s-1}(R), g(u)=-b_{2} \partial_{x} \Lambda^{-2} u^{2}$ and $W=\Lambda$. Then we will verify that $Q(u)$ and $g(u)$ satisfy conditions (I)-(III). We cite several conclusions presented in [11].

Lemma 3.1 The operator $Q(u)=\left(\alpha_{0}+b_{1} u\right) \partial_{x}$ with $\phi \in H^{s}(R)\left(s>\frac{3}{2}\right)$ belongs to $G\left(H^{s-1}, 1, \alpha\right)$.

Lemma 3.2 For $u, z, w \in H^{s}(R)$ with $s>\frac{3}{2}, Q(\phi) \in L\left(H^{s}(R), H^{s-1}(R)\right)$, it holds that

$$
\|(Q(u)-Q(z)) w\|_{H^{s-1}} \leq \rho_{1}\|u-z\|_{H^{s-1}}\|w\|_{H^{s}} .
$$

Lemma 3.3 For $u, z \in H^{s}(R)$ and $w \in H^{s-1}(R)\left(s>\frac{3}{2}\right)$, it holds that $\bar{Q}(u)=[\Lambda,(1-$ $\left.\left.b_{1} u\right) \partial_{x}\right] \Lambda^{-1} \in L\left(H^{s-1}\right)$ and

$$
\|(\bar{Q}(u)-\bar{Q}(z)) w\|_{H^{s-1}} \leq \rho_{2}\|u-z\|_{H^{s}}\|w\|_{H^{s-1}} .
$$

Lemma 3.4 ([20]) Let $r$ and $q$ be real numbers such that $-r<q \leq r$. Then

$$
\begin{aligned}
& \left\|u_{1} u_{2}\right\|_{H^{q}} \leq c\left\|u_{1}\right\|_{H^{r}}\left\|u_{2}\right\|_{H^{q}} \quad \text { if } r>\frac{1}{2}, \\
& \left\|u_{1} u_{2}\right\|_{H^{r+q-\frac{1}{2}}} \leq c\left\|u_{1}\right\|_{H^{r}}\left\|_{2}\right\|_{H^{q}} \quad \text { if } r<\frac{1}{2} .
\end{aligned}
$$

Lemma 3.5 Let $u, z \in H^{s}(R)$ with $s>\frac{3}{2}$ and $g(u)=-b_{2} \partial_{x} \Lambda^{-2} u^{2}$. Then $g$ is bounded on bounded sets in $H^{s}$ and satisfies

$$
\begin{aligned}
& \|g(u)-g(z)\|_{H^{s}} \leq \rho_{3}\|u-z\|_{H^{s}}, \\
& \|g(u)-g(z)\|_{H^{s-1}} \leq \rho_{4}\|u-z\|_{H^{s-1}} .
\end{aligned}
$$

Proof For $s>\frac{3}{2}$, we have $\|u\|_{L^{\infty}} \leq c\|u\|_{H^{s}}$ and $\|u\|_{H^{s-1}} \leq c\|u\|_{H^{s}}$. Applying the algebra property of $H^{s}(R)$ and Lemma 3.4, we get

$$
\begin{aligned}
\|g(u)-g(z)\|_{H^{s}} & \leq\left|b_{2}\right|\left\|\partial_{x} \Lambda^{-2}\left(u^{2}-z^{2}\right)\right\|_{H^{s}} \\
& \leq c\left\|u^{2}-z^{2}\right\|_{H^{s-1}} \\
& \leq c\|u-z\|_{H^{s-1}} \\
& \leq \rho_{3}\|u-z\|_{H^{s}},
\end{aligned}
$$

which completes the proof of (10). Similarly, we get (11).

Now we give the following theorem. 
Theorem 3.6 Let $u_{0}(x) \in H^{s}(R)$ with $s>\frac{3}{2}$. There exists $T>0$ depending on $\left\|u_{0}\right\|_{H^{s}(R)}$ such that problem (6) or (7) has a unique solution $u(t, x) \in C\left([0, T) ; H^{s}(R)\right) \cap C^{1}\left([0, T) ; H^{s-1}(R)\right)$.

Proof Using Lemmas 3.1-3.3 and 3.5, we know that conditions (I), (II) and (III) hold. Applying the Kato theorem, we find that problem (6) or (7) has a unique local solution

$$
u=u(t, x) \in C\left([0, T) ; H^{s}(R)\right) \cap C^{1}\left([0, T) ; H^{s-1}(R)\right)
$$

where $T>0$ depends on $\left\|u_{0}\right\|_{H^{s}}$.

Remark 3.7 Let $T>0$ be described in Theorem 3.6. Using the Sobolev embedding theorem, we ensure the boundedness of solution $u(t, x)$ to problem (7) in the domain $[0, T) \times R$. Namely, provided that $u_{0} \in H^{s}(R)$ with $s>\frac{3}{2}$, we have $\|u\|_{L^{\infty}(R)} \leq M_{T}$, where $M_{T}$ is a positive constant.

\section{$4 L^{1}$ Stability of the local solution for problem (6)}

Letting $f^{\prime}(u)=\alpha_{0}+b_{1} u, \Psi(t, x, u)=b_{2} \Lambda^{-2} \partial_{x} u^{2}$ in the first equation of (7), we get

$$
\left\{\begin{array}{l}
u_{t}+f^{\prime}(u) u_{x}+\Psi(t, x, u)=0 \\
u(0, x)=u_{0}(x)
\end{array}\right.
$$

Assume that $u_{1}(t, x)$ and $u_{2}(t, x)$ are solutions of problem (12) in the domain $[0, T) \times R$ with initial functions $u_{10}(x)$ and $u_{20}(x) \in H^{s}(R)\left(s>\frac{3}{2}\right)$. Here we give several lemmas.

Lemma 4.1 Let $u(t, x)$ be the solution of problem (12) and $u_{0}(x) \in H^{s}(R)$ with $s>\frac{3}{2}$. Then $\|\Psi(t, x, u)\|_{L^{\infty}(R)} \leq M_{T}$ and

$$
\|\Psi(t, x, u)\|_{L^{\infty}(R)} \leq c M_{T}^{2}
$$

where positive constant $c$ depends on $b_{2}, \beta,\left\|u_{0}\right\|_{L^{\infty}}$.

Proof We have

$$
\begin{aligned}
|\Psi(t, x, u)| & =\left|b_{2} \Lambda^{-2} \partial_{x} u^{2}\right| \\
& \leq\left|b_{2}\right|\left|\frac{1}{2 \beta} \int_{R} e^{-\frac{1}{\beta}|x-y|} \operatorname{sign}(y-x) u^{2} d y\right| \\
& \leq c M_{T}^{2} .
\end{aligned}
$$

Applying Remark 3.7 and the integral $\int_{R} e^{-\frac{1}{\beta}|x-y|} d y=2 \beta$, we complete the proof.

Lemma 4.2 Assume that $u_{1}(t, x)$ and $u_{2}(t, x)$ are solutions of problem (12) in the domain $[0, T) \times R$ with initial functions $u_{10}(x)$ and $u_{20}(x) \in H^{s}(R)\left(s>\frac{3}{2}\right)$, respectively. Then

$$
\int_{-\infty}^{+\infty}\left|\Psi\left(t, x, u_{1}\right)-\Psi\left(t, x, u_{2}\right)\right| d x \leq c \int_{-\infty}^{+\infty}\left|u_{1}-u_{2}\right| d x
$$

where $c>0$ depends on $b_{2}, \beta,\left\|u_{10}\right\|_{L^{\infty}(R)},\left\|u_{20}\right\|_{L^{\infty}(R)}$ and $T$. 
Proof Using the property of the operator $\Lambda^{-2}$ and Remark 3.7, we get

$$
\begin{aligned}
& \int_{-\infty}^{+\infty}\left|\Psi\left(t, x, u_{1}\right)-\Psi\left(t, x, u_{2}\right)\right| d x \\
& \quad=\int_{-\infty}^{+\infty}\left|b_{2} \partial_{x} \Lambda^{-2}\left(u_{1}^{2}-u_{2}^{2}\right)\right| d x \\
& \quad \leq c \int_{-\infty}^{+\infty} d x \int_{-\infty}^{+\infty}\left|e^{-\frac{1}{\beta}|x-y|} \operatorname{sign}(y-x)\left(u_{1}^{2}-u_{2}^{2}\right)\right| d y \\
& \quad \leq c \int_{-\infty}^{+\infty}\left|u_{1}^{2}-u_{2}^{2}\right| d x \int_{-\infty}^{+\infty} e^{-\frac{1}{\beta}|x-y|} d y \\
& \quad \leq c \int_{-\infty}^{+\infty}\left|u_{1}-u_{2}\right| d x,
\end{aligned}
$$

in which we apply the Tonelli theorem to complete the proof.

We introduce a function $\delta(\sigma)$ which is infinitely differential on $(-\infty,+\infty)$ and $\delta(\sigma) \geq$ $0, \delta(\sigma) \equiv 0$ for $|\sigma| \geq 1, \int_{-\infty}^{+\infty} \delta(\sigma) d \sigma=1$. Let $\delta_{\varepsilon}(\sigma)=\delta\left(\varepsilon^{-1} \sigma\right) / \varepsilon$, where $\varepsilon$ is an arbitrary positive constant. It is found that $\delta_{\varepsilon}(\sigma) \in C_{0}^{\infty}(-\infty,+\infty)$ and

$$
\left\{\begin{array}{l}
\delta_{\varepsilon}(\sigma) \geq 0, \quad \delta_{\varepsilon}(\sigma)=0 \quad \text { for }|\sigma| \geq \varepsilon \\
\left|\delta_{\varepsilon}(\sigma)\right| \leq \frac{c}{\varepsilon}, \quad \int_{-\infty}^{+\infty} \delta_{\varepsilon}(\sigma) d \sigma=1 .
\end{array}\right.
$$

Let the function $\phi(x)$ be defined and locally integrable on $(-\infty,+\infty)$. Set $\phi^{\varepsilon}(x)$ to denote the approximation function of $\phi(x)$ as

$$
\phi^{\varepsilon}(x)=\frac{1}{\varepsilon} \int_{-\infty}^{+\infty} \delta\left(\frac{x-y}{\varepsilon}\right) \phi(y) d y
$$

We call $x_{0}$ a Lebesgue point of the function $\phi(x)$ if

$$
\lim _{\varepsilon \rightarrow 0} \frac{1}{\varepsilon} \int_{x_{0}-\varepsilon}^{x_{0}+\varepsilon}\left|\phi(x)-\phi\left(x_{0}\right)\right| d x=0 .
$$

At any Lebesgue point $x_{0}$, we get

$$
\lim _{\varepsilon \rightarrow 0} \phi^{\varepsilon}\left(x_{0}\right)=\phi\left(x_{0}\right) .
$$

Since the set of points which are not Lebesgue points of $\phi(x)$ has measure zero, we have $\phi^{\varepsilon}(x) \rightarrow \phi(x)$ as $\varepsilon \rightarrow 0$ almost everywhere.

For any $T_{0} \in[0, T)$, we denote the band $\left\{(t, x):\left[0, T_{0}\right] \times R\right\}$ by $\pi_{T_{0}}$. Let $K_{r}=\{x:|x| \leq r\}$ and

$$
\Pi=\left\{(t, x, \tau, y):\left|\frac{t-\tau}{2}\right| \leq \varepsilon, \rho \leq \frac{t+\tau}{2} \leq T_{0}-\rho,\left|\frac{x-y}{2}\right| \leq \varepsilon,\left|\frac{x+y}{2}\right| \leq r-\rho\right\},
$$

where $r>0, \rho>0$.

Lemma 4.3 ([19]) Let the function $\phi(t, x)$ be bounded and measurable in cylinder $\left[0, T_{0}\right] \times$ $K_{r}$. If for any $\rho \in\left(0, \min \left[r, T_{0}\right]\right)$ and any $\varepsilon \in(0, \rho)$, the function

$$
V_{\varepsilon}=\frac{1}{\varepsilon^{2}} \iiint \int_{\Pi}|\phi(t, x)-\phi(\tau, y)| d t d x d \tau d y
$$


satisfies

$$
\lim _{\varepsilon \rightarrow 0} V_{\varepsilon}=0 .
$$

Lemma 4.4 ([19]) If $\frac{\partial \Phi(\phi)}{\partial \phi}$ is bounded, then the function $H\left(\phi_{1}, \phi_{2}\right)=\operatorname{sign}\left(\phi_{1}-\phi_{2}\right)\left(\Phi\left(\phi_{1}\right)-\right.$ $\left.\Phi\left(\phi_{2}\right)\right)$ satisfies the Lipschitz condition in $\phi_{1}$ and $\phi_{1}$.

We state the concept of a characteristic cone. Let $T$ be described in Theorem 3.6 and $\|\phi\|_{L^{\infty}(R)} \leq M_{T}$. For any $T_{0} \in[0, T)$ and $R_{0}>0$, we define

$$
N>\max _{(t, x) \in\left[0, T_{0}\right] \times K_{R_{0}}}\left|f^{\prime}(\phi)\right| .
$$

Let $\Omega$ represent the cone $\left\{(t, x):|x| \leq R_{0}-N t, 0 \leq t \leq T_{1}=\min \left(T_{0}, R_{0} N^{-1}\right)\right\}$ and $S_{\tau}$ designate the cross-section of the cone $\Omega$ by the plane $t=\tau, \tau \in\left[0, T_{1}\right]$.

Lemma 4.5 Let $u(t, x)$ be the solution of problem (12) on $\pi_{T_{0}}, q(t, x) \in C_{0}^{\infty}\left(\pi_{T_{0}}\right)$. It holds that

$$
\begin{gathered}
\iint_{\pi_{T_{0}}}\left\{|u-k| q_{t}+\operatorname{sign}(u-k)[f(u)-f(k)] q_{x}\right. \\
-\operatorname{sign}(u-k) q(t, x) \Psi(t, x, u)\} d t d x=0,
\end{gathered}
$$

where $k$ is an arbitrary constant.

Proof Suppose that $F(u)$ is a twice differential function. Multiplying the first equation of problem (12) by $F^{\prime}(u) q(t, x)$ and integrating over $\pi_{T_{0}}$, we get

$$
\iint_{\pi_{T_{0}}}\left\{F^{\prime}(u) q u_{t}+F^{\prime}(u) q f^{\prime}(u) u_{x}+F^{\prime}(u) q \Psi(t, x, u)\right\} d t d x=0 .
$$

The application of the method of integration by parts yields

$$
\iint_{\pi_{T_{0}}} F^{\prime}(u) q u_{t} d t d x=-\iint_{\pi_{T_{0}}} F^{\prime}(u) q_{t} d t d x
$$

Since

$$
\left(\int_{k}^{u} F^{\prime}(z) f^{\prime}(z) d z\right)^{\prime}=F^{\prime}(u) f^{\prime}(u) u_{x}
$$

we have

$$
\begin{aligned}
\iint_{\pi_{T_{0}}} F^{\prime}(u) f^{\prime}(u) u_{x} q d t d x= & -\iint_{\pi_{T_{0}}}\left(\int_{k}^{u} F^{\prime}(z) f^{\prime}(z) d z\right) q_{x} d t d x \\
= & -\iint_{\pi_{T_{0}}}\left\{F^{\prime}(u)[f(u)-f(k)]\right. \\
& \left.-\int_{k}^{u} F^{\prime \prime}(z)[f(z)-f(k)] d z\right\} q_{x} d t d x .
\end{aligned}
$$


Substitute Eqs. (18) and (19) into Eq. (17). Let $F^{\varepsilon}(u)$ be an approximation of the function $F(u)=|u-k|$. When $\varepsilon \rightarrow 0, F^{\varepsilon}(u) \rightarrow F(u)$, we obtain Eq. (16).

Set function $q(t, x) \in C_{0}^{\infty}\left(\pi_{T_{0}}\right), q(t, x) \equiv 0$ outside the cylinder $\varpi=\{(t, x)\}=\left[\rho, T_{0}-\right.$ $2 \rho] \times K_{r-2 \rho}$, where $K_{r-2 \rho}=\{|x|:|x| \leq r-2 \rho\}, r>0,0<2 \rho<\min \left(T_{0}, r\right)$. Now we give the proof of the local stability for problem (6) or (7).

Theorem 4.6 Assume that $u_{1}(t, x)$ and $u_{2}(t, x)$ are two local strong solutions of problem (6) or (7) with initial data $u_{0}(x), v_{0}(x) \in L^{1}(R) \cap H^{s}(R)\left(s>\frac{3}{2}\right)$, respectively. Let $T>0$ be the maximum existence time of $u_{1}(t, x)$ and $u_{2}(t, x)$. For any $t \in[0, T)$, it holds that

$$
\left\|u_{1}(t, x)-u_{2}(t, x)\right\|_{L^{1}} \leq e^{c t}\left\|u_{10}(x)-u_{20}(x)\right\|_{L^{1}}
$$

where $c$ is a positive constant depending on $\left\|u_{10}\right\|_{L^{\infty}(R)}$ and $\left\|u_{20}\right\|_{L^{\infty}(R)}$.

Proof We define

$$
P(t, x, \tau, y)=q\left(\frac{t+\tau}{2}, \frac{x+y}{2}\right) \delta_{\varepsilon}\left(\frac{t-\tau}{2}, \frac{x-y}{2}\right)=q(\cdots) \lambda_{\varepsilon}(\dot{:}),
$$

in which $(\cdots)=\left(\frac{t+\tau}{2}, \frac{x+y}{2}\right),(\dot{:})=\left(\frac{t-\tau}{2}, \frac{x-y}{2}\right)$. Thus, we obtain

$$
P_{t}+P_{\tau}=q_{t}(\cdots) \lambda_{\varepsilon}(\dot{:}), \quad P_{x}+P_{y}=q_{x}(\cdots) \lambda_{\varepsilon}(\dot{\vdots})
$$

Using Lemma 4.5 and the Kruzkov's device of doubling the variables [19], we get

$$
\begin{aligned}
& \iiint \int_{\pi_{T_{0}} \times \pi_{T_{0}}}\left\{\left|u_{1}(t, x)-u_{2}(\tau, y)\right| P_{t}+\operatorname{sign}\left(u_{1}(t, x)-u_{2}(\tau, y)\right)\right. \\
& \quad \times\left[f\left(u_{1}(t, x)\right)-f\left(u_{2}(\tau, y)\right)\right] P_{x}-\operatorname{sign}\left(u_{1}(t, x)-u_{2}(\tau, y)\right) \\
& \left.\quad \times \Psi\left(t, x, u_{1}(t, x)\right) P\right\} d t d x d \tau d y=0 .
\end{aligned}
$$

Similarly, we have

$$
\begin{aligned}
& \iiint \int_{\pi_{T_{0}} \times \pi_{T_{0}}}\left\{\left|u_{2}(\tau, y)-u_{1}(t, x)\right| P_{\tau}+\operatorname{sign}\left(u_{2}(\tau, y)-u_{1}(t, x)\right)\right. \\
& \quad \times\left[f\left(u_{2}(\tau, y)\right)-f\left(u_{1}(t, x)\right)\right] P_{y}-\operatorname{sign}\left(u_{2}(\tau, y)-u_{1}(t, x)\right) \\
& \left.\quad \times \Psi\left(\tau, y, u_{2}(\tau, y)\right) P\right\} d t d x d \tau d y=0 .
\end{aligned}
$$

Adding (21) and (22), we obtain

$$
\begin{aligned}
0 \leq & \iiint \int_{\pi_{T_{0}} \times \pi_{T_{0}}}\left\{\left|u_{1}(t, x)-u_{2}(\tau, y)\right| q_{t} \lambda_{\varepsilon}\right. \\
& +\operatorname{sign}\left(u_{1}(t, x)-u_{2}(\tau, y)\right) \\
& \left.\times\left[f\left(u_{1}(t, x)\right)-f\left(u_{2}(\tau, y)\right)\right] q_{x} \lambda_{\varepsilon}\right\} d t d x d \tau d y
\end{aligned}
$$




$$
\begin{aligned}
& +\mid \iiint \int_{\pi_{T_{0}} \times \pi_{T_{0}}}\left\{\operatorname{sign}\left(u_{1}(t, x)-u_{2}(\tau, y)\right)\right. \\
& \left.\times\left[\Psi\left(t, x, u_{1}(t, x)\right)-\Psi\left(\tau, y, u_{2}(\tau, y)\right)\right] q \lambda_{\varepsilon}\right\} d t d x d \tau d y \mid
\end{aligned}
$$

We note that the first two terms of the integrand of (23) have the form

$$
G_{\varepsilon}=G\left(t, x, \tau, y, u_{1}(t, x), u_{2}(\tau, y)\right) \lambda_{\varepsilon}(\dot{:}),
$$

where $G$ satisfies the Lipschitz condition in all its variables. Then

$$
\begin{aligned}
& \iiint \int_{\pi_{T_{0}} \times \pi_{T_{0}}} G_{\varepsilon} d t d x d \tau d y \\
& =\iiint \int_{\pi_{T_{0}} \times \pi_{T_{0}}} G\left(t, x, \tau, y, u_{1}(t, x), u_{2}(\tau, y)\right) \lambda_{\varepsilon} d t d x d \tau d y \\
& =\iiint \int_{\pi_{T_{0}} \times \pi_{T_{0}}}\left\{G\left(t, x, \tau, y, u_{1}(t, x), u_{2}(\tau, y)\right)\right. \\
& \left.\quad-G\left(t, x, t, x, u_{1}(t, x), u_{2}(t, x)\right)\right\} \lambda_{\varepsilon} d t d x d \tau d y \\
& \quad+\iiint \int_{\pi_{T_{0}} \times \pi_{T_{0}}} G\left(t, x, t, x, u_{1}(t, x), u_{2}(t, x)\right) \lambda_{\varepsilon} d t d x d \tau d y \\
& =A_{11}(\varepsilon)+A_{12} .
\end{aligned}
$$

As $G_{\varepsilon}=0$ outside the region $\Pi$, applying the estimate $\left|\lambda_{\varepsilon}(:)\right| \leq \frac{c}{\varepsilon^{2}}$ and Lemma 4.4 , we get

$$
\left|A_{11}(\varepsilon)\right| \leq c\left[\varepsilon+\frac{1}{\varepsilon^{2}} \iiint \int_{\Pi}\left|u_{1}(t, x)-u_{2}(\tau, y)\right| d t d x d \tau d y\right]
$$

where $c$ is a positive constant independent of $\varepsilon$. Using Lemma 4.3, we know $A_{11}(\varepsilon) \rightarrow 0$ as $\varepsilon \rightarrow 0$.

For the term $A_{12}$, we substitute $t=\eta_{1}, \frac{t-\tau}{2}=\mu_{1}, x=\eta_{2}, \frac{x-y}{2}=\mu_{2}$. Combining with the identity

$$
\int_{-\varepsilon}^{\varepsilon} \int_{-\infty}^{+\infty} \lambda_{\varepsilon}\left(\mu_{1}, \mu_{2}\right) d \mu_{1} d \mu_{2}=1
$$

we obtain

$$
\begin{aligned}
A_{12}= & 2^{2} \iint_{\pi_{T_{0}} \times \pi_{T_{0}}} G\left(\eta_{1}, \eta_{2}, \eta_{1}, \eta_{2}, u_{1}\left(\eta_{1}, \eta_{2}\right), u_{2}\left(\eta_{1}, \eta_{2}\right)\right) \\
& \times\left(\int_{-\varepsilon}^{\varepsilon} \int_{-\infty}^{+\infty} \lambda_{\varepsilon}\left(\mu_{1}, \mu_{2}\right) d \mu_{1} d \mu_{2}\right) d \eta_{1} d \eta_{2} \\
= & 4 \iint_{\pi_{T_{0}}} G\left(t, x, t, x, u_{1}(t, x), u_{2}(t, x)\right) d t d x .
\end{aligned}
$$

Thus, we have

$$
\lim _{\varepsilon \rightarrow 0} \iiint \int_{\pi_{T_{0}} \times \pi_{T_{0}}} G_{\varepsilon} d t d x d \tau d y=4 \iint_{\pi_{T_{0}}} G\left(t, x, t, x, u_{1}(t, x), u_{2}(t, x)\right) d t d x .
$$


Similarly, the integrand of the third term in (23) can be represented as

$$
\begin{aligned}
B_{\varepsilon} & =\operatorname{sign}\left(u_{1}(t, x)-u_{2}(\tau, y)\right)\left[\Psi\left(t, x, u_{1}(t, x)\right)-\Psi\left(\tau, y, u_{2}(\tau, y)\right)\right] q \lambda_{\varepsilon} \\
& =B\left(t, x, \tau, y, u_{1}(t, x), u_{2}(\tau, y)\right) \lambda_{\varepsilon}(:) .
\end{aligned}
$$

Then

$$
\begin{aligned}
& \iiint \int_{\pi_{T_{0}} \times \pi_{T_{0}}} B_{\varepsilon} d t d x d \tau d y \\
& =\iiint \int_{\pi_{T_{0} \times \pi_{T_{0}}}}\left\{B\left(t, x, \tau, y, u_{1}(t, x), u_{2}(\tau, y)\right)\right. \\
& \left.\quad-B\left(t, x, t, x, u_{1}(t, x), u_{2}(t, x)\right) \lambda_{\varepsilon}\right\} d t d x d \tau d y \\
& \quad+\iiint \int_{\pi_{T_{0}} \times \pi_{T_{0}}} B\left(t, x, t, x, u_{1}(t, x), u_{2}(t, x)\right) \lambda_{\varepsilon} d t d x d \tau d y \\
& =A_{21}(\varepsilon)+A_{22} .
\end{aligned}
$$

Applying Lemma 4.4, we get

$$
\left|A_{21}(\varepsilon)\right| \leq c\left[\varepsilon+\frac{1}{\varepsilon^{2}} \iiint \int_{\Pi}\left|u_{1}(t, x)-u_{2}(\tau, y)\right| d t d x d \tau d y\right] .
$$

By using Lemma 4.3, it yields $A_{21}(\varepsilon) \rightarrow 0$ as $\varepsilon \rightarrow 0$. Repeating the steps as before, we have

$$
A_{22}=4 \iint_{\pi_{T_{0}}} B\left(t, x, t, x, u_{1}(t, x), u_{2}(t, x)\right) d t d x
$$

From (23) to (27), we get

$$
\begin{gathered}
\iint_{\pi_{T_{0}}}\left\{\left|u_{1}(t, x)-u_{2}(t, x)\right| q_{t}+\operatorname{sign}\left(u_{1}(t, x)-u_{2}(t, x)\right)\left[f\left(u_{1}\right)-f\left(u_{2}\right)\right] q_{x}\right\} d t d x \\
\quad+\left|\iint_{\pi_{T_{0}}} \operatorname{sign}\left(u_{1}(t, x)-u_{2}(t, x)\right)[\Psi(t, x, \phi)-\Psi(t, x, \psi)] q d t d x\right| \geq 0
\end{gathered}
$$

Set

$$
l(t)=\int_{-\infty}^{+\infty}\left|u_{1}(t, x)-u_{2}(t, x)\right| d x
$$

and

$$
v_{\varepsilon}(\sigma)=\int_{-\infty}^{\sigma} \delta_{\varepsilon}(z) d z
$$

Take two numbers $\rho, \tau \in\left(0, T_{1}\right)$ and $\rho<\tau$. In (28), we let

$$
q(t, x)=\left[v_{\varepsilon}(t-\rho)-v_{\varepsilon}(t-\tau)\right] \chi(t, x), \quad \varepsilon<\min \left(\rho, T_{1}-\tau\right),
$$


in which

$$
\chi(t, x)=\chi_{\theta}(t, x)=1-v_{\theta}\left(|x|+N t-R_{0}+\theta\right),
$$

where $\theta$ is a small positive constant and $\chi(t, x)=0$ outside the cone $\Omega$. When $\theta \rightarrow 0$, $R_{0} \rightarrow+\infty$, we observe that $\chi_{\theta} \rightarrow 1$. By the definition of the number $N$, we have

$$
0=\chi_{t}+N\left|\chi_{x}\right| \geq \chi_{t}+N \chi_{x}, \quad(t, x) \in \Omega
$$

According to (28)-(32), we get

$$
\begin{aligned}
& \iint_{\pi_{T_{0}}}\left\{\left|u_{1}(t, x)-u_{2}(t, x)\right|\left[\delta_{\varepsilon}(t-\rho)-\delta_{\varepsilon}(t-\tau)\right] \chi_{\theta}(t, x)\right\} d t d x \\
& \quad+\int_{0}^{T_{1}} d t \int_{-\infty}^{+\infty}\left\{\left|\Psi\left(t, x, u_{1}\right)-\Psi\left(t, x, u_{2}\right)\right|\right. \\
& \left.\quad \times\left[v_{\varepsilon}(t-\rho)-v_{\varepsilon}(t-\tau)\right] \chi_{\theta}(t, x)\right\} d x \geq 0 .
\end{aligned}
$$

For (33), sending $\theta \rightarrow 0, R_{0} \rightarrow+\infty$ combining with Lemma 4.2, we obtain

$$
\int_{0}^{T_{1}}\left[\delta_{\varepsilon}(t-\rho)-\delta_{\varepsilon}(t-\tau)\right] l(t) d t+c \int_{0}^{T_{1}}\left[v_{\varepsilon}(t-\rho)-v_{\varepsilon}(t-\tau)\right] l(t) d t \geq 0,
$$

where $c$ is independent of $\varepsilon$.

Applying the properties of the function $\delta_{\varepsilon}$ for $\varepsilon \leq \min \left(\rho, T_{1}-\rho\right)$, we get

$$
\begin{aligned}
\left|\int_{0}^{T_{1}} \delta_{\varepsilon}(t-\rho) l(t)-l(\rho) d t\right| & =\left|\int_{0}^{T_{1}} \delta_{\varepsilon}(t-\rho)[l(t)-l(\rho)] d t\right| \\
& \leq \frac{c}{\varepsilon} \int_{\rho-\varepsilon}^{\rho+\varepsilon}|l(t)-l(\rho)| d t .
\end{aligned}
$$

Consequently,

$$
\int_{0}^{T_{1}} \delta_{\varepsilon}(t-\rho) l(t) d t \rightarrow l(\rho) \quad \text { as } \varepsilon \rightarrow 0
$$

Now set

$$
K(\rho)=\int_{0}^{T_{1}} v_{\varepsilon}(t-\rho) l(t) d t=\int_{0}^{T_{1}} d t \int_{-\infty}^{t-\rho} \delta_{\varepsilon}(\sigma) l(t) d \sigma .
$$

It is seen that

$$
K^{\prime}(\rho)=-\int_{0}^{T_{1}} \delta_{\varepsilon}(t-\rho) l(t) d t .
$$

Letting $\varepsilon \rightarrow 0$, we derive

$$
K^{\prime}(\rho) \rightarrow-l(\rho)
$$


and

$$
K(\rho) \rightarrow K(0)-\int_{0}^{\rho} l(t) d t, \quad K(\tau) \rightarrow K(0)-\int_{0}^{\tau} l(t) d t .
$$

Hence, we get

$$
K(\rho)-K(\tau) \rightarrow \int_{\rho}^{\tau} l(t) d t \quad \text { as } \varepsilon \rightarrow 0
$$

Applying (34)-(36), we obtain the inequality

$$
l(\rho)+c \int_{\rho}^{\tau} l(t) d t \geq l(\tau)
$$

As $\rho \rightarrow 0, \tau \rightarrow t$, we get the following result:

$$
\begin{aligned}
& \int_{-\infty}^{+\infty}\left|u_{1}(0, x)-u_{2}(0, x)\right| d x+c \int_{0}^{t} d t \int_{-\infty}^{+\infty}\left|u_{1}(t, x)-u_{2}(t, x)\right| d x \\
& \quad \geq \int_{-\infty}^{+\infty}\left|u_{1}(t, x)-u_{2}(t, x)\right| d x
\end{aligned}
$$

Finally, we complete the proof of Theorem 4.6 by using the Gronwall inequality.

\section{Competing interests}

The authors declare that they have no competing interests.

\section{Authors' contributions}

Both authors contributed to each part of this study equally and approved the final version of the manuscript.

\section{Author details}

'School of Science, Southwest University of Science and Technology, Mianyang, 621000, China. ${ }^{2}$ School of Economics, Peking University, Beijing, 100871, China.

\section{Acknowledgements}

This work is supported by the National Natural Science Foundation of China (11471263).

Received: 8 July 2015 Accepted: 6 September 2015 Published online: 18 September 2015

\section{References}

1. Degasperis, A, Procesi, M: Asymptotic integrability. In: Symmetry and Perturbation Theory, pp. 23-37. World Scientific, Singapore (1999)

2. Degasperis, A, Holm, DD, Hone, ANW: A new integrable equation with peakon solution. Theor. Math. Phys. 2, 1463-1474 (2002)

3. Lenells, J. Traveling wave solutions of the Degasperis-Procesi equation. J. Math. Anal. Appl. 306, 72-82 (2005)

4. Coclite, GM, Karlsen, KH: Periodic solutions of the Degasperis-Procesi equation: well-posedness and asymptotics. J. Funct. Anal. 268, 1053-1077 (2015)

5. Coclite, GM, Karlsen, $\mathrm{KH}$, Risebro, NH: Numerical schemes for computing discontinuous solutions of the Degasperis-Procesi equation. IMA J. Numer. Anal. 28, 80-105 (2007)

6. Escher, J, Liu, Y, Yin, ZY: Global weak solutions and blow-up structure for the Degasperis-Procesi equation. J. Funct. Anal. 241, 457-485 (2006)

7. Lenells, J: Conservation laws of the Camassa-Holm equation. J. Phys. A 38, 869-880 (2005)

8. Cavalcanti, MM, Domingos Cavalcanti, VN, Komornik, V, Rodrigues, JH: Global well-posedness and exponential decay rates for a KdV-Burgers equation with indefinite damping. Ann. Inst. Henri Poincaré, Anal. Non Linéaire 31, 1079-1100 (2014)

9. Hirayama, H: Local well-posedness for the periodic higher order KdV type equations. Nonlinear Differ. Equ. Appl. 19, 677-693 (2012)

10. Wazwaz, AM: New kinds of solitons and periodic solutions to the generalized KdV equation. Numer. Methods Partial Differ. Equ. 23, 247-255 (2007)

11. Rodriguez-Blanco, G: On the Cauchy problem for the Camassa-Holm equation. Nonlinear Anal. 46, 309-327 (2001)

12. Degasperis, A, Holm, DD, Hone, ANW: A new integral equation with peakon solutions. Theor. Math. Phys. 133, 1461-1477 (2002) 
13. Coclite, GM, Karlsen, KH: On the well-posedness of the Degasperis-Procesi equation. J. Funct. Anal. 233, 60-91 (2006)

14. Escher, J, Yin, Z: Well-posedness, blow-up phenomena and global solutions for the $b$-equation. J. Reine Angew. Math. $624,51-80$ (2008)

15. Lai, SY, Yan, HB, Chen, HJ, Wang, J: The stability of local strong solutions for a shallow water equation. J. Inequal. Appl. 2014, 410 (2014)

16. Gu, F, Lu, YG, Zhang, Q: Global solutions to one-dimensional shallow water magnetohydrodynamic equations. J. Math. Anal. Appl. 401, 714-723 (2013)

17. Mutlubas, ND: Local well-posedness and wave breaking results for periodic solutions of a shallow water equation for waves of moderate amplitude. Nonlinear Anal. 97, 145-154 (2014)

18. Liu, XX, Yin, ZY: Local well-posedness and stability of solitary waves for the two-component Dullin-Gottwald-Holm system. Nonlinear Anal. 88, 1-15 (2013)

19. Kruzkov, SN: First order quasi-linear equations in several independent variables. Math. USSR Sb. 10, $217-243$ (1970)

20. Kato, T: Quasi-linear equations of evolution with applications to partial differential equations. In: Spectral Theory and Differential Equations. Lecture Notes in Math., vol. 448, pp. 25-70 (1975)

\section{Submit your manuscript to a SpringerOpen ${ }^{\circ}$ journal and benefit from:}

- Convenient online submission

Rigorous peer review

- Immediate publication on acceptance

- Open access: articles freely available online

- High visibility within the field

- Retaining the copyright to your article 